\title{
Fasciocutaneous Free Flaps for Reconstruction of Hypopharyngeal Defects
}

\author{
Cesare Piazza, MD (D; Francesca Del Bon, MD; Alberto Paderno, MD; Alberto Grammatica, MD; \\ Nausica Montalto, MD; Valentina Taglietti, MD; Piero Nicolai, MD
}

\begin{abstract}
Objectives/Hypothesis: Different reconstructive options are available for defects following total laryngectomy (TL) and circumferential $(\mathrm{CH})$ or partial hypopharyngectomy $(\mathrm{PH})$. We evaluated the flap success, pharyngocutaneous fistula, and pharyngoesophageal stenosis rates in two groups of patients treated by different policies.

Study Design: Comparison between two cohorts of patients treated by TL with $\mathrm{PH} / \mathrm{CH} \pm$ cervical esophagectomy and reconstructed according to different strategies.

Methods: Group A (historical) was composed of 89 patients reconstructed by pectoralis major myocutaneous (PMMC), radial forearm (RF), and anterolateral thigh (ALT) flaps. A salivary bypass stent (SBPS) was not routinely applied and left in place for a maximum of 14 days. Forty-four (49\%) patients received preoperative radiotherapy/chemoradiotherapy (RT/CRT). Group B (prospective) included 105 patients reconstructed by RF or ALT with long-lasting SBPS left in place for a maximum of 45 days. Sixty-one (59\%) received preoperative RT/CRT.

Results: In group A, flap failure occurred in four (4\%) cases, and all were managed by PMMC. We encountered 22 $(26 \%)$ fistulas and $14(16 \%)$ stenoses. In group B, flap failure occurred in six (6\%) cases and was managed by PMMC. We encountered seven $(7 \%)$ fistulas and three $(3 \%)$ stenoses. Comparing complications among the two groups, we encountered a statistically significant difference in favor of group B for both fistula $(P<.001)$ and stenosis $(P=.001)$. We did not evidence any significant difference in terms of flap success rate.
\end{abstract}

Conclusions: First-line application of RF and ALT free flaps with long-lasting SBPS in reconstruction after PH/CH allows obtaining reduced incidences of both fistula and stenosis.

Key Words: Hypopharyngectomy, reconstruction, free flap, fistula, stenosis.

Level of Evidence: 4.

Laryngoscope, 127:2731-2737, 2017

\section{INTRODUCTION}

Reconstruction following laryngo-hypopharyngectomy for hypopharyngeal squamous cell carcinoma (HSCC) remains a challenge in head and neck surgical practice. The ideal reconstructive technique for such defects, possibly involving the cervical esophagus, should be that with the lowest ensuing morbidity (especially in terms of fistula and stricture rates) and mortality, as well as the most rapid and efficient swallowing rehabilitation. The minimum amount of healthy mucosa necessary to primarily close the neopharynx without excessive risk of pharyngocutaneous fistula (PCF) and/or postoperative stenosis is considered to be at least $2.5 \mathrm{~cm}{ }^{1}$ However, even in naïve HSCC to be treated by total laryngectomy (TL) with partial hypopharyngectomy $(\mathrm{PH})$, this must be considered more the exception than the rule. Moreover, the contemporary tendency to

From the Department of Otorhinolaryngology-Head and Neck Surgery, University of Brescia, Brescia, Italy.

Editor's Note: This Manuscript was accepted for publication April 27, 2017.

The authors have no funding, financial relationships, or conflicts of interest to disclose.

Send correspondence to Cesare Piazza, MD, Department of Otorhinolaryngology-Head and Neck Surgery, University of Brescia, Spedali Civili of Brescia, Piazza Spedali Civili 1, 25123 Brescia, Italy. E-mail: ceceplaza@libero.it

DOI: 10.1002/lary.26705 primarily treat HSCC by nonsurgical organ preservation strategies generally hampers the possibility to primarily close a swollen and devascularized hypopharyngeal mucosa even when the amount of residual tissue should exceed $2.5 \mathrm{~cm}$.

Currently, fasciocutaneous free flaps, in particular radial forearm $(\mathrm{RF})^{2-9}$ and anterolateral thigh (ALT), ${ }^{5,9-17}$ are considered among the main options for such a reconstructive purpose, especially considering the high rates of PCF and stenosis generally observed after pectoralis major myocutaneous (PMMC) pedicled flap, ${ }^{5,7,9,15,18-21}$ and the non-negligible complication rate associated with a jejunum free flap. ${ }^{5,7,9,15,22-38}$

The aim of the present report was to describe our reconstructive policy and technique after $\mathrm{TL}$ and $\mathrm{PH}$ or circumferential hypopharyngectomy $(\mathrm{CH})$, with or without cervical esophagectomy (CE), analyzing flap failure, complications, $\mathrm{PCF}$, and pharyngoesophageal stricture (PES) rates observed in a partially retrospective, partially prospective clinical series of HSCC treated in the same tertiary academic institution.

\section{MATERIALS AND METHODS}

This study is based on the comparison between two groups of patients submitted to TL and $\mathrm{PH}$ or $\mathrm{CH}$ with/without $\mathrm{CE}$ for pT3-T4 and rypT2-T4 HSCC followed by primary reconstruction at the Department of Otorhinolaryngology Head and Neck 


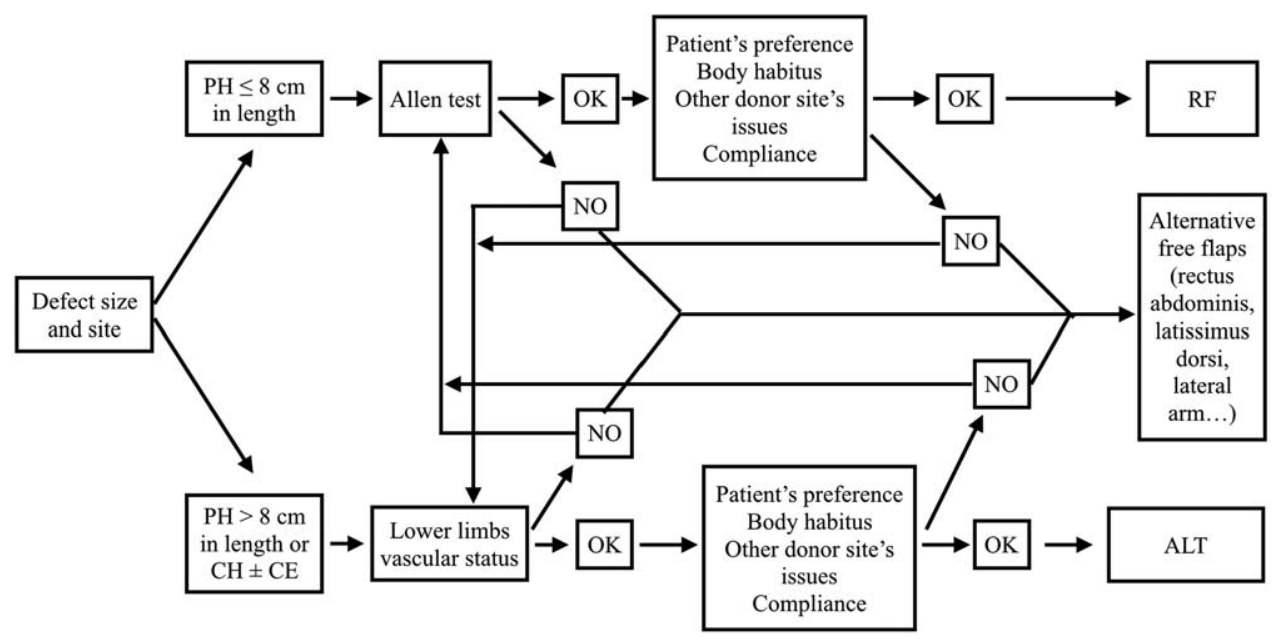

Fig. 1. Flowchart applied since January 2009 as reconstructive algorithm in group $B$. ALT = anterolateral thigh free flap; CE $=$ cervical esophagectomy; $\mathrm{CH}$ = circumferential hypopharyngectomy; $\mathrm{PH}=$ partial hypopharyngectomy; $\mathrm{RF}=$ radial forearm free flap.

Surgery, University of Brescia, Brescia, Italy. Group A was evaluated retrospectively in December 2008 and gave us the basis for changing our reconstructive policy detailed in Figure 1, which was then systematically applied from January 2009 onward in group B, prospectively evaluated (Table I).

\section{Group A}

Group A included 89 patients treated between January 1996 and December 2008 in which the reconstructive policy encompassed use of either PMMC, RF, or ALT flaps. Furthermore, a salivary bypass stent (SBPS) was placed only in patients previously treated by radiotherapy (RT)/chemoradiotherapy (CRT), not fixed to the skin, and removed after no more than 15 days from surgery (Table I). No primary tracheoesophageal puncture was ever performed.

\section{Group B}

Due to the relatively high incidence of PCF, PES, and SBPS migration observed in group A, in January 2009 we changed our reconstructive policy with the purpose of reducing the incidence of these complications. All patients with primary or recurrent $\mathrm{HSCC}$ requiring $\mathrm{PH}$ or $\mathrm{CH}$ with/without $\mathrm{CE}$ were prospectively enrolled in group B. In this group of patients we introduced the first-line use of fasciocutaneous free flaps (RF and ALT), routinely associated with a long-lasting (45 days) SBPS fixed to the chin skin, and standardized antibiotic prophylaxis with sulbactam-ampicillin (or quinolones in case of penicillin allergy) and metronidazole (after RT/CRT failure). Primary endpoints to be evaluated were the incidence of PCF, PES, and flap failure that were compared to those observed in the historical cohort. This group included 105 patients treated and evaluated between January 2009 and June 2015 (Tables I and II).

\section{Surgical Technique}

All reconstructive procedures in group B were carried out using the same technique by the same senior surgeon (c.P.). Before starting reconstruction, precise measurements of the surgical defects were taken (including distance between base of tongue and proximal esophageal stump, width of base of tongue, and width of the esophageal stump). A vertical slit $1.5 \mathrm{~cm}$ in length was made at the level of the anterior wall of the esophageal stump. An SBPS (Montgomery Salivary Bypass Tube; Boston Medical Products, Shrewsbury, MA) was then inserted in the surgical defect, with its proximal end at the level of the base of tongue and the distal one cut to lie about $4 \mathrm{~cm}$ below

TABLE I.

Characteristics of the Two Cohorts.

Group A

Group B

$\begin{array}{ll}\text { Treatment period } & \text { January 1996-December } 2008 \\ \text { Type of evaluation } & \text { Retrospective } \\ \text { No. of patients } & 89 \\ \text { Age, yr } & \text { Range, 17-83; mean, } 61 \\ \text { Gender } & 79 \text { males, } 10 \text { females } \\ \text { Previous RT/CRT } & 44(49 \%) \\ \text { First line reconstruction } & \text { PMMC, ALT, RF } \\ \text { Salivary bypass stent } & \text { Only in irradiated patients, left for } 15 \text { days } \\ \text { Antibiotic prophylaxis } & \text { According to patient characteristics and } \\ & \text { risk factors profile }\end{array}$

January 2009-June 2015

Prospective

105

Range, 35-85; mean, 66

89 males, 16 females

$61(59 \%)$

ALT, RF

In all patients, left for 45 days

Standardized, nonirradiated patients: ampicillinsulbactam for 15 days; irradiated patients: ampicillin-sulbactam + metronidazole for 15 days

$\mathrm{ALT}=$ anterolateral thigh; $\mathrm{CRT}=$ chemoradiotherapy; $\mathrm{PMMC}=$ pectoralis major myocutaneous; $\mathrm{RF}=$ radial forearm; $\mathrm{RT}=$ radiotherapy. 


\begin{tabular}{|c|c|c|c|}
\hline \multicolumn{4}{|c|}{$\begin{array}{c}\text { TABLE II. } \\
\text { Comparison of Different Variables Between Groups A and B. }\end{array}$} \\
\hline & Group A & Group B & $P$ \\
\hline \multicolumn{4}{|l|}{ Age } \\
\hline$\leq 65$ years & $71 \%$ & $45 \%$ & \\
\hline$>65$ years & $29 \%$ & $65 \%$ & $<.001$ \\
\hline \multicolumn{4}{|l|}{ Gender } \\
\hline Male & $89 \%$ & $85 \%$ & \\
\hline Female & $11 \%$ & $15 \%$ & NS \\
\hline \multicolumn{4}{|l|}{ Stage } \\
\hline$|-|||$ & $11 \%$ & $16 \%$ & \\
\hline IV & $89 \%$ & $84 \%$ & NS \\
\hline \multicolumn{4}{|c|}{ Previous RT/CRT } \\
\hline No & $51 \%$ & $41 \%$ & \\
\hline Yes & $49 \%$ & $59 \%$ & NS \\
\hline \multicolumn{4}{|c|}{ Surgical defect } \\
\hline $\mathrm{PH}$ & $80 \%$ & $76 \%$ & \\
\hline $\mathrm{CH}$ & $20 \%$ & $24 \%$ & NS \\
\hline \multicolumn{4}{|c|}{ Cervical esophagectomy } \\
\hline No & $81 \%$ & $79 \%$ & \\
\hline Yes & $19 \%$ & $21 \%$ & NS \\
\hline
\end{tabular}

$\mathrm{CH}=$ circumferential hypopharyngectomy; CRT = chemoradiotherapy; $\mathrm{NS}=$ not statistically significant; $\mathrm{PH}=$ partial hypopharyngectomy; RT = radiotherapy.

the edge of the esophageal resection. A nasogastric feeding tube (NGFT) was always placed inside the SBPS (Fig. 2A). The stent was fixed through the base of tongue to the chin skin by a nonreabsorbable stitch (Fig. 2B). At this point in the intervention, we started harvesting the planned free flap (chosen on the base of length of the surgical defect, available recipient vessels in the neck, body habitus, comorbidities, vascular abnormalities, and patient preferences). After pedicle ligation, the free flap was transposed into the neck and sutured in a horseshoe shape with the lateral edges of the residual posterior remnant of the hypopharynx in case of $\mathrm{PH}$ (Fig. 2C), or directly to the prevertebral fascia in case of $\mathrm{CH}$ (Fig. 2D,E). No complete tubulization of the flap was ever attempted to get the largest neopharynx possible with the smallest skin paddle harvested. When reconstruction was performed by ALT, the flap was sutured using a two-layer closure, with the fascia lata wrapped around the neopharynx to reinforce the suture lines (Fig. 2F). Free flap viability was checked by pin-pricking the skin monitor externalized above the stoma in case of ALT or through a stab wound in the middle of the cervical skin flap in case of RF (Fig. 2G). Antibiotic prophylaxis with ampicillin-sulbactam (associated with metronidazole after RT/CRT failure) was routinely started during surgery and protracted for a minimum of 2 weeks after surgery. The NGFT was usually removed 12 days after surgery, and a liquid-to-soft diet started with the SBPS in situ. Tracheoesophageal puncture was never attempted, primarily due to concomitant level VI and VII lymph node dissection and ensuing separation of trachea and esophagus below the sternal notch. The SBPS was removed through the mouth at 45 days after surgery in the outpatient clinic.

\section{Statistical Analysis}

In both groups, we evaluated the flap success rate, incidence of complications, and PCF and PES occurrence to quantify the benefit of the new surgical strategy.
The following variables were evaluated by univariate analysis using Fisher exact or $\chi^{2}$ tests to identify factors predictive of complications (flap failure, PCF, and PES): group of patients (A vs. B), preoperative RT/CRT, extension of the defect ( $\mathrm{PH}$ vs. $\mathrm{CH}$ vs. $\mathrm{CE}$ ), and type of reconstruction (fasciocutaneous free flaps vs. PMMC). Multivariate logistic regression analysis was used to further evaluate the association between those variables and complications. A two-tailed $P<.05$ was considered statistically significant. Statistical analysis was carried out using Stata software version 13.0 (StataCorp, College Station, TX).

\section{RESULTS}

\section{Group A}

In group A, $18(20 \%)$ patients were submitted to $\mathrm{CH}$ and $71(80 \%)$ to $\mathrm{PH}$. Seventeen patients (19\%) had surgical resection extended to the CE. Primary reconstructive options encompassed PMMC in 39 (44\%), RF in $46(52 \%)$, and ALT in $4(4 \%)$ patients. SBPS was used only in 44 previously irradiated patients and left in place up to its dislocation $(n=10)$, excessive pain/discomfort $(n=8)$, or for a maximum of 15 days $(n=26)$. One $(1 \%)$ perioperative death occurred after a major bleeding 25 days after surgery. Flap failure occurred in four (4\%) patients (one RF, three PMMC) and was secondarily managed by PMMC in all cases. One of these revised patients developed a $\mathrm{PCF}$, whereas another presented a PES, both of which were treated conservatively.

In successfully primarily reconstructed patients, we encountered $22(26 \%)$ PCFs after a mean of 6 days (range, 3-8 days); 17 were cured by medical treatment with local curettage and antibiotic therapy in association or not with hyperbaric oxygen therapy (HOT), and eight needed revision surgery with direct suture $(\mathrm{n}=5)$ or PMMC $(\mathrm{n}=3)$. Fourteen (16\%) patients experienced late PES, which was managed by endoscopic dilatations in 11 patients (single procedure in six, multiple in four patients), a second free flap in one, and permanent gastrostomy tube in three cases. Further surgical complications were encountered in $22(25 \%)$ patients: nine $(10 \%)$ wound dehiscence managed by medical treatment, six $(7 \%)$ bleedings requiring revision, $4(4 \%)$ chylous leaks (managed by compressive dressing in 3 cases and requiring PMMC in one), and three (3\%) neck abscesses requiring drainage.

In this group, PCF occurred in 16 (24\%) patients submitted to $\mathrm{PH}$ and in six $(33 \%)$ patients submitted to $\mathrm{CH}$. Of these, four (23\%) had CE extension. No statistically significant difference was demonstrated. Considering nonirradiated patients and those previously treated by RT/CRT, we observed PCF in nine (20\%) and $13(32 \%)$ cases, respectively. Again, we did not evidence statistically significant difference.

Regarding PES, 11 (16\%) and three (17\%) patients had received $\mathrm{PH}$ and $\mathrm{CH}$, respectively, whereas seven $(16 \%)$ had not received previous treatments and seven (17\%) failed at RT/CRT. In addition, the PES rate was not influenced by the extension of resection and previous RT/CRT.

Comparing the use of PMMC with fasciocutaneous free flaps in terms of functional outcomes and complications, we found a significant difference in the PES rate 

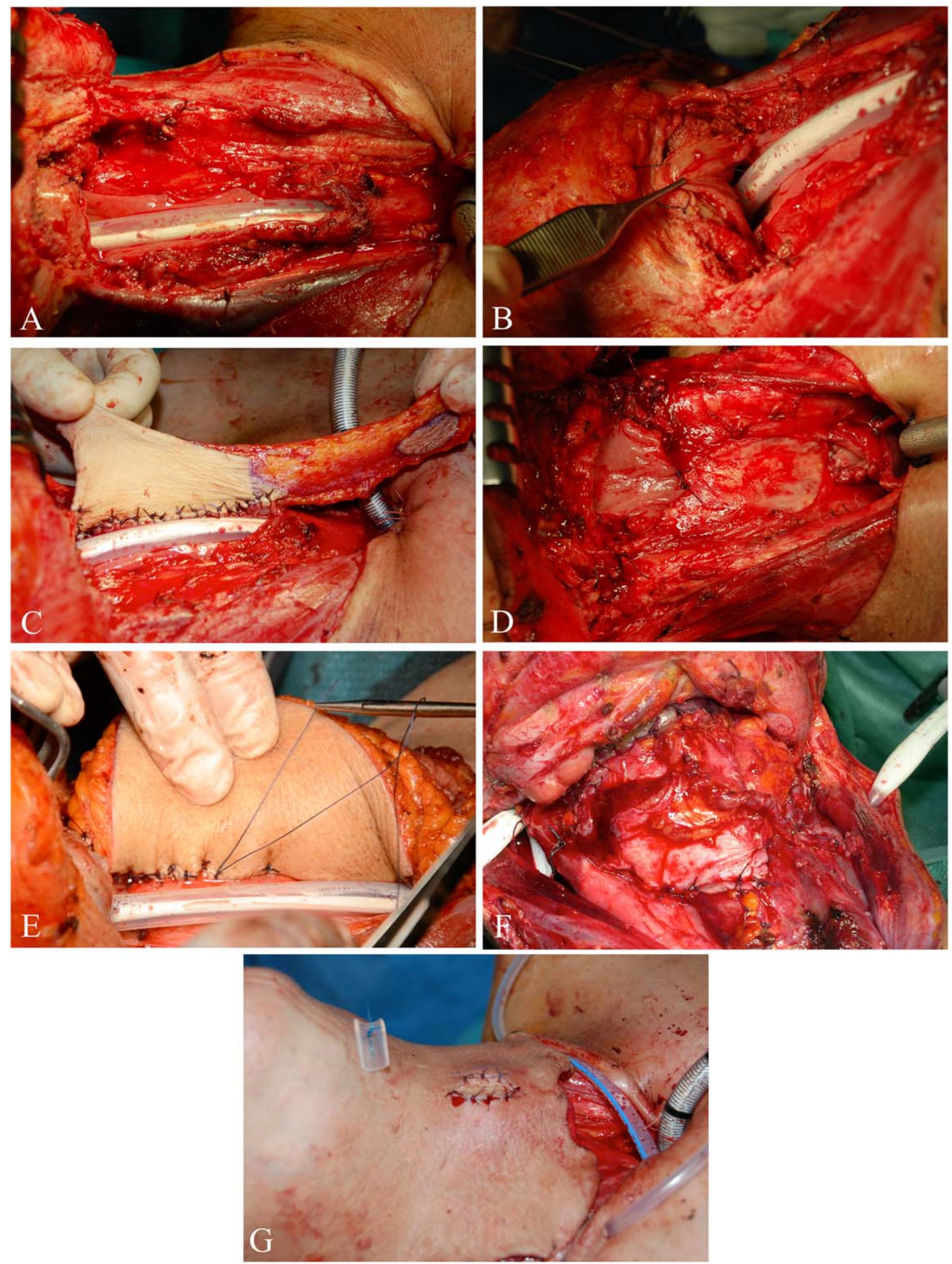

Fig. 2. (A) Intraoperative view showing the SBPS (containing the NGFT) inserted after TL with PH. (B) Intraoperative detail showing the nonreabsorbable stitch going through the base of tongue (up to the chin skin) placed to prevent postoperative migration of the SBPS. (C) Reconstruction of a $\mathrm{PH}$ defect using an RF free flap sutured to the posterior remnant of the hypopharyngeal mucosa. (D) Intraoperative view after TL with $\mathrm{CH}$. The mucosal margin of the posterior wall of the oropharynx and the posterior part of the esophageal stump are sutured to the prevertebral fascia. (E) Reconstruction of a $\mathrm{CH}$ defect with an ALT free flap. The lateral edge of the skin paddle is being sutured to the prevertebral fascia. (F) Surgical field after insetting of an ALT free flap. The fascia lata has been wrapped around the neopharynx to reinforce the pharyngeal closure. $(G)$ Intraoperative view before skin closure showing the skin monitor of a RF free flap sutured to the cervical skin and the nonreabsorbable stitch fixing the SBPS to the chin skin. ALT = anterolateral thigh; $\mathrm{CH}=$ circumferential hypopharyngectomy; NGFT = nasogastric feeding tube; $\mathrm{PH}=$ partial hypopharyngectomy; RF = radial forearm free flap; SBPS = salivary bypass stent; TL = total laryngectomy. [Color figure can be viewed in the online issue, which is available at www.laryngoscope.com.] 
( $28 \%$ vs. $8 \%, P=.012$ ) in favor of free flaps, with no difference in $\mathrm{PCF}(31 \%$ vs. $20 \%, P=.243)$ or flap failure rates ( $8 \%$ vs. $2 \%, P=.221)$.

\section{Group B}

In group B, $25(24 \%)$ patients were submitted to $\mathrm{CH}$ and $80(76 \%)$ to $\mathrm{PH}$. CE was associated in $22(21 \%)$ cases. Reconstructive options encompassed $\mathrm{RF}$ in 46 $(44 \%)$ and ALT in $59(56 \%)$ patients. An SBPS was placed in all patients and removed after 45 days, with stent migration requiring early removal in only two $(2 \%)$ patients due to rupture of the stitch at the level of the chin skin. There were no perioperative deaths. Flap failure occurred in six (6\%) cases and was secondarily managed by PMMC in all cases. Among these patients, one later developed PCF and was managed by conservative treatment. Considering all viable flap transfers, we encountered seven (7\%) PCFs after a mean of 20 days (range, 14-28 days); two were cured by medical treatment with local curettage and antibiotic therapy, and five needed revision surgery with direct suture of the PCF. Three (3\%) patients experienced late PES (successfully managed by a single endoscopic dilatation in two and three procedures in one patient). Further surgical complications were encountered in 27 (26\%) patients: 18 (17\%) wound dehiscence (managed by local medications in 16 cases, HOT in one, and PMMC in one), five (5\%) bleedings requiring revision, two $(2 \%)$ cervical spondylodiscitis managed by medical treatment, one (1\%) venous thrombosis of the microvascular anastomosis managed by surgical revision without subsequent flap failure, one (1\%) cervical abscess managed by local medications, and one $(1 \%)$ chylous leak treated with compressive dressing.

PCF occurred in five (7\%) patients submitted to $\mathrm{PH}$ and in two $(9 \%)$ submitted to $\mathrm{CH}(P=.759)$. Three $(8 \%)$ PCFs occurred in previously untreated patients and four $(7 \%)$ after RT/CRT $(P=.908)$. Moreover, PCF occurred in five $(12 \%) \mathrm{RF}$ and in two $(4 \%) \operatorname{ALT}(P=.127)$ cases.

Distribution of PES was as follows: one $(1 \%) \mathrm{PH}$ versus two (8\%) $\mathrm{CH}$, two (5\%) RF versus one (2\%) ALT, and one $(2 \%)$ naïve versus two $(3 \%)$ post-RT/CRT. The PES rate was not significantly influenced by the abovementioned variables.

\section{Group A Versus Group B}

Comparing complications between groups A and B, we found a significant difference in favor of group $B$ for both PCF $(P<.001)$ and $\operatorname{PES}(P=.001)$. On the contrary, flap failure rate did not show any significant difference between the two groups $(P=.702)$.

Multivariate analysis confirmed the statistically significant difference between the two groups in terms of PCF ( $P=.016)$, whereas other variables analyzed were not significant (age, preoperative RT/CRT, $\mathrm{CH}$ vs. $\mathrm{PH}$, $\mathrm{CE}$, and use of free flaps). On the other hand, considering the same variables in relation to PES rate, only the use of free flaps was related to a significant difference $(P=$ $.015)$, whereas none of the other variables were significant between groups.

\section{DISCUSSION}

Since CRT became the primary treatment for most advanced laryngeal and hypopharyngeal squamous cell carcinomas, surgery has usually been reserved for locally advanced and persistent/recurrent diseases. In such cases, primary closure of the neopharynx at the end of TL with $\mathrm{CH}$ or $\mathrm{PH}$ may not be possible, or may carry a high risk for PCF and PES due both to the hypovascularized residual tissue after $\mathrm{RT} / \mathrm{CRT}^{39}$ and a scarce amount of healthy mucosa after extensive resections for advanced tumors. In consideration of the poor long-term survival of these patients, it is mandatory to employ a reliable one-stage reconstruction, granting at the same time good functional outcomes, limited morbidity, and rapid rehabilitation of swallowing with early resumption of oral feeding. A number of techniques have been described to achieve these outcomes, including PMMC, gastric pull-up, free jejunal transposition, and fasciocutaneous free flaps. However, careful evaluation of pros and cons of each technique is mandatory and should take into consideration individual patient characteristics and specific risk factors. ${ }^{9}$ It is well known that patients with head and neck cancer frequently have a long history of tobacco and alcohol consumption associated with significant comorbidities. For this reason, major reconstructive procedures requiring laparotomy/laparoscopy may deplete their functional reserve and further impact their general status. In particular, free jejunal transposition is frequently associated with postoperative ileus, potentially leading to major complications, prolonged recovery, and longer hospital stay. In this view, although functional results are, in the best case scenario, comparable with those obtained using fasciocutaneous free flaps, there is a higher perioperative mortality frequently related to abdominal complications, ${ }^{5,7,9,15,22-38}$ even when considering large series of patients. ${ }^{40}$ On the other hand, pedicled flaps such as the PMMC are more suited as an alternative to free flaps in patients with general or local contraindications for microsurgery. ${ }^{5,7,9,15,18-21}$ In this view, a study by Lee et al. ${ }^{41}$ showed a lower rate of medical and pulmonary complications in PMMC but an increase of surgical ones. In fact, the thickness and weight of such a pedicled flap can lead to loss of suture tightness with subsequent onset of PCF or PES due to excessive bulk. This has been demonstrated in a meta-analysis by Chao et al. ${ }^{42}$ who reported significantly higher rates of PCF $(24.7 \%$ vs. $8.9 \%, P<$ $.001)$ and requirement for revision surgery (11.3\% vs. $5.5 \%, P=.04)$ in PMMC in comparison with fasciocutaneous free flaps.

In our series, we observed a significant reduction of PCF and PES from group A to group B: $26 \%$ vs. $7 \%(P<$ $.001)$ and $18 \%$ vs. $3 \%(P=.001)$, respectively, confirming the advantages of our policy based on the first-line use of fasciocutaneous free flaps together with long-lasting SBPS. These data are in agreement with those published by $\mathrm{Yu}$ et al. showing $\mathrm{PCF}$ in $9 \%$ and $\mathrm{PES}$ in $6 \%$ in a series of 114 patients submitted to TL and reconstruction with ALT. ${ }^{13}$ Similarly, Lopez et al. reported PCF in $9 \%$ after reconstruction with RF or ALT and SBPS placement (16\% with RF and $3 \%$ with ALT). ${ }^{43}$ This is also confirmed 
by smaller series employing RF or ALT together with SBPS for hypopharyngeal reconstruction. In particular, Varvares et al. ${ }^{44}$ published a retrospective study on 20 patients who had undergone reconstruction of the hypopharynx and CE using an RF and SBPS with an overall rate of PCF of $20 \%$ and PES of $10 \%$. Finally, no patient in the series reported by Murray et al. ${ }^{11}$ developed PCF using an ALT with a SBPS, even if this series included only 14 subjects.

The use of SBPS was first introduced to divert salivary flow in patients who developed fistulae. As already mentioned, Lopez et al., ${ }^{43}$ Varvares et al., ${ }^{44}$ and Murray et al. ${ }^{11}$ described the use of fasciocutaneous free flaps in conjunction with the SBPS to minimize exposure of the anastomotic suture line to saliva during the early healing phase. This may help in reducing the incidence of PCFs, anastomotic leaks, and PES as also confirmed in our series. A study by Punthakee et al. ${ }^{45}$ further reinforced this hypothesis showing (by univariate analysis) a significantly lower PCF rate in patients receiving SBPS ( $22.4 \%$ vs. $7.4 \%, P=.048)$. Our choice to leave the SBPS in place for 45 days is related to our previous experience where we observed development of PCFs until the 40th postoperative day. Fixation of the SBPS to the chin skin has been demonstrated to reduce postoperative discomfort related to the presence of a foreign body at the level of the base of tongue and prevents its dislocation into the distal esophagus or stomach, as reported in the literature. ${ }^{11,43,44,46}$ This occurred in only $2 \%$ of patients of our prospective cohort.

Regarding surgical technique, there is no clear indication on the ideal flap configuration for reconstruction of $\mathrm{CH}$. However, it is important to not underestimate the impact of this aspect on outcomes and complications. In this view, the majority of authors do not detail their surgical technique or describe the employment of fasciocutaneous flaps in a tubular fashion when reconstructing $\mathrm{CH}$ defects, with highly variable outcomes, going up to $53 \%$ for $\mathrm{PCF}$ and $36 \%$ for PES. ${ }^{9,47,48}$ In our experience, suturing in a horseshoe shape the lateral edges of the free flap to the prevertebral fascia for $\mathrm{CH}$ leads to a large neopharynx, with low PES rates and reduced amount of tissue needed for reconstruction, thus favoring the wound closure at the level of the donor site. In fact, the amount of transferred tissue was maximum 14 $\mathrm{cm}$ in length and $8 \mathrm{~cm}$ in width, allowing direct suture at the ALT donor site and minimizing the size of skin graft in the RF (usually applied for smaller defects). Favorable outcomes have also been confirmed by other authors applying this technique, with PCF and PES rates not exceeding $13 \%$ and $5 \%$, respectively. ${ }^{7,43}$

Furthermore, our results do not show any significant difference in terms of surgical outcomes when considering irradiated and nonirradiated patients. This may be related to the favorable influence of bringing wellvascularized tissue to a less-vascularized surgical bed, thus improving wound healing. ${ }^{49}$ Moreover, use of ALT in such circumstances allows employing the fascia lata as a second layer for the external reinforcement of the suture line. When needed, this flap can also be harvested with a double skin paddle to reconstruct part of the cervical skin if heavily damaged by RT/CRT and/or previous surgery.

\section{CONCLUSION}

Our study demonstrates that fasciocutaneous free flaps represent a reliable and successful reconstructive option after TL extended to the hypopharynx and cervical esophagus, regardless of the primary or salvage settings in which reconstruction is needed. Their use in conjunction with a long-lasting SBPS allowed for significant reduction in the incidence of PCF and PES in the prospective cohort of patients herein considered.

\section{BIBLIOGRAPHY}

1. Hui Y, Wei WI, Yuen PW, Lam LK, Ho WK. Primary closure of pharyngeal remnant after total laryngectomy and partial pharyngectomy: how much residual mucosa is sufficient? Laryngoscope 1996;106:490-494.

2. Azizzadeh B, Yafai S, Rawnsley JD, et al. Radial forearm free flap pharyngoesophageal reconstruction. Laryngoscope 2001;111:807-810.

3. Disa JJ, Pusic AL, Hidalgo DA, Cordeiro PG. Microvascular reconstruction of the hypopharynx: defect classification, treatment algorithm, and functional outcome based on 165 consecutive cases. Plast Reconstr Surg 2003;111:652-660.

4. Scharpf J, Esclamado RM. Reconstruction with radial forearm flaps after ablative surgery for hypopharyngeal cancer. Head Neck 2003;25:261266.

5. Clark JR, Gilbert R, Irish J, Brown D, Neligan P, Gullane PJ. Morbidity after flap reconstruction of hypopharyngeal defects. Laryngoscope 2006; 116:173-181.

6. Andrades P, Pehler SF, Baranano CF, Magnuson JS, Carroll WR, Rosenthal EL. Fistula analysis after radial forearm free flap reconstruction of hypopharyngeal defects. Laryngoscope 2008;118:1157-1163.

7. Hong JW, Jeong HS, Lew DH, et al. Hypopharyngeal reconstruction using remnant narrow pharyngeal wall as omega-shaped radial forearm free flap. J Craniofac Surg 2009;20:1334-1340.

8. Yang CC, Lee JC, Wu KC, Chang SH. Voice and speech outcomes with radial forearm free flap-accompanied phonation tube after total pharyngolaryngectomy of hypopharyngeal cancer. Acta Otolaryngol 2011;131: 847-851.

9. Piazza C, Taglietti V, Nicolai P. Reconstructive options after total laryngectomy with subtotal or circumferential hypopharyngectomy and cervical esophagectomy. Curr Opin Otolaryngol Head Neck Surg 2012;20:77-88.

10. Genden EM, Jacobson AS. The role of the anterolateral thigh flap for pharyngoesophageal reconstruction. Arch Otolaryngol Head Neck Surg 2005;131:796-799.

11. Murray DJ, Gilbert RW, Vesely MJ, et al. Functional outcomes and donor site morbidity following circumferential pharyngoesophageal reconstruction using an anterolateral thigh flap and salivary bypass tube. Head Neck 2007;29:147-154.

12. Sagar B, Marres HA, Hartman EH. Hypopharyngeal reconstruction with an anterolateral thigh flap after laryngopharyngeal resection: results of a retrospective study on 20 patients. J Plast Reconstr Aesthet Surg 2010;63:970-975.

13. Yu P, Hanasono MM, Skoracki RJ, et al. Pharyngoesophageal reconstruction with the anterolateral thigh flap after total laryngopharyngectomy. Cancer 2010;116:1718-1724.

14. Spyropoulou GA, Lin PY, Chien CY, Kuo YR, Jeng SF. Reconstruction of the hypopharynx with the anterolateral thigh flap: defect classification, method, tips, and outcomes. Plast Reconstr Surg 2011;127:161-172.

15. Chan YW, Ng RW, Liu LH, Chung HP, Wei WI. Reconstruction of circumferential pharyngeal defects after tumour resection: reference or preference. J Plast Reconstr Aesthet Surg 2011;64:1022-1028.

16. Tan NC, Yeh MC, Shih HS, Nebres RP, Yang JC, Kuo YR. Single free anterolateral thigh flap for simultaneous reconstruction of composite hypopharyngeal and external neck skin defect after head and neck cancer ablation. Microsurgery 2011;31:524-528.

17. Tan NC, Shih HS, Chen CC, Chen YC, Lin PY, Kuo YR. Distal skin paddle as a monitor for buried anterolateral thigh flap in pharyngoesophageal reconstruction. Oral Oncol 2012;48:249-252.

18. Morshed K, Szymanski M, Golabek W. Reconstruction of the hypopharynx with U-shaped pectoralis major myocutaneous flap after total pharyngolaryngectomy. Eur Arch Otorhinolaryngol 2005;262:259-262.

19. Saussez S, Cuno A, Urbain F, Chantrain G, Lequeux T. Reconstruction of circumferential oro- and hypopharyngeal defects with U-shaped pectoralis major myocutaneous flap. Otolaryngol Head Neck Surg 2006;134: 823-829.

20. Benazzo M, Bertino G, Occhini A, Spasiano R, Gatti P. Functional outcomes in patients reconstructed with flaps following surgery for hypopharyngeal cancer. Acta Otorhinolaryngol Ital 2006;26:127-132.

21. Xiao Q, Hu GH, Zhong SX, Qian Y, Zeng Q, Hong SL. Reconstruction of hypopharynx and cervical oesophagus for treatment of advanced 
hypopharyngeal carcinoma and recurrent laryngeal carcinoma. Asian $J$ Surg 2010;33:14-19.

22. Triboulet JP, Mariette C, Chevalier D, Amrouni H. Surgical management of carcinoma of the hypopharynx and cervical esophagus: analysis of 209 cases. Arch Surg 2001;136:1164-1170.

23. Oniscu GC, Walker WS, Sanderson R. Functional results following pharyngolaryngooesophagectomy with free jejunal graft reconstruction. Eur $J$ Cardiothorac Surg 2001;19:406-410.

24. Benazzo M, Occhini A, Rossi V, Aresi G, Alessiani M. Jejunum free flap in hypopharynx reconstruction: case series. BMC Cancer 2002;2:13.

25. Wadsworth JT, Futran N, Eubanks TR. Laparoscopic harvest of the jejunal free flap for reconstruction of hypopharyngeal and cervical esophageal defects. Arch Otolaryngol Head Neck Surg 2002;128:1384-1387.

26. Bova R, Goh R, Poulson M, Coman WB. Total pharyngolaryngectomy for squamous cell carcinoma of the hypopharynx: a review. Laryngoscope 2005; 115:864-869.

27. Lewin JS, Barringer DA, May AH, et al. Functional outcomes after circumferential pharyngoesophageal reconstruction. Laryngoscope 2005; $115: 1266-1271$.

28. Nyquist GG, Hier MP, Dionisopoulos T, Black MJ. Stricture associated with primary tracheoesophageal puncture after pharyngolaryngectomy and free jejunal interposition. Head Neck 2006;28:205-209.

29. Yu P, Lewin JS, Reece GP, Robb GL. Comparison of clinical and functional outcomes and hospital costs following pharyngoesophageal reconstruction with the anterolateral thigh free flap versus the jejunal flap. Plast Reconstr Surg 2006;117:968-974.

30. Disa JJ, Pusic AL, Mehrara BJ. Reconstruction of the hypopharynx with the free jejunum transfer. $J$ Surg Oncol 2006;94:466-470.

31. Sarukawa S, Sakuraba M, Kimata Y, et al. Standardization of free jejunum transfer after total pharyngolaryngoesophagectomy. Laryngoscope 2006;116:976-981.

32. Hanson RP, Chow TK, Feehan E, Eadie PA, Timon CT, Keogh S. Analysis of functional results and quality of life following free jejunal flaps for reconstruction after upper aerodigestive neoplastic resection: the St James's experience. J Plast Reconstr Aesthet Surg 2007;60:577-582.

33. Dubsky PC, Stift A, Rath T, Kornfehl J. Salvage surgery for recurrent carcinoma of the hypopharynx and reconstruction using jejunal free tissue transfer and pectoralis major muscle pedicled flap. Arch Otolaryngol Head Neck Surg 2007;133:551-555.

34. Ikeguchi M, Miyake T, Matsunaga T, et al. Free jejunal graft reconstruction after resection of neck cancers: our surgical technique. Surg Today 2009;39:925-928

35. Zhao D, Gao X, Guan L, et al. Free jejunal graft for reconstruction of defects in the hypopharynx and cervical esophagus following the cancer resections. J Gastrointest Surg 2009;13:1368-1372.
36. Moradi P, Glass GE, Atherton DD, et al. Reconstruction of pharyngolar yngectomy defects using the jejunal free flap: a 10-year experience from a single reconstructive center. Plast Reconstr Surg 2010:126:1960-1966.

37. Kadota H, Fukushima J, Nakashima T, et al. Comparison of salvage and planned pharyngolaryngectomy with jejunal transfer for hypopharyngeal carcinoma after chemoradiotherapy. Laryngoscope 2010;120:1103-1108.

38. Numajiri T, Sowa Y, Nishino K, et al. Double vascular anastomosis in the neck for reliable free jejunal transfer. Br J Oral Maxillofac Surg 2010; 48:511-514.

39. Dedivitis RA, Aires FT, Cernea CR, Brandao LG. Pharyngocutaneous fistula after total laryngectomy: systematic review of risk factors. Head Neck 2015;37:1691-1697.

40. Perez-Smith D, Wagels M, Theile DR. Jejunal free flap reconstruction of the pharyngolaryngectomy defect: 368 consecutive cases. J Plast Reconstr Aesthet Surg 2013;66:9-15.

41. Lee T, Chung C, Chang Y, Kim J. Comparison of clinical and functional outcomes using pectoralis major and cutaneous free flaps for hypopharyngeal squamous cell carcinoma. Arch Plast Surg 2015;42:608-613.

42. Chao JW, Spector JA, Taylor EM, et al. Pectoralis major myocutaneous flap versus free fasciocutaneous flap for reconstruction of partial hypopharyngeal defects: what should we be doing? J Reconstr Microsurg 2015;31:198-204

43. Lopez F, Obeso S, Camporro D, Fueyo A, Suarez C, Llorente JL. Outcomes following pharyngolaryngectomy with fasciocutaneous free flap reconstruction and salivary bypass tube. Laryngoscope 2013;123:591-596.

44. Varvares MA, Cheney ML, Gliklich RE, et al. Use of the radial forearm fasciocutaneous free flap and montgomery salivary bypass tube for pharyngoesophageal reconstruction. Head Neck 2000;22:463-468.

45. Punthakee X, Zaghi S, Nabili V, Knott PD, Blackwell KE. Effects of salivary bypass tubes on fistula and stricture formation. JAMA Facial Plast Surg 2013;15:219-225.

46. Leon X, Quer M, Burgues J. Montgomery salivary bypass tube in the reconstruction of the hypopharynx. Cost-benefit study. Ann Otol Rhinol Laryngol 1999;108:864-868.

47. Chen WF, Chang KP, Chen CH, Shyu VB, Kao HK. Outcomes of anterolateral thigh flap reconstruction for salvage laryngopharyngectomy for hypopharyngeal cancer after concurrent chemoradiotherapy. PLoS One 2013;8:e53985.

48. Zelken JA, Kang CJ, Huang SF, Liao CT, Tsao CK. Refinements in flap design and inset for pharyngoesophageal reconstruction with free thigh flaps. Microsurgery 2017;37:112-118.

49. Paderno A, Piazza C, Bresciani L, Vella R, Nicolai P. Microvascular head and neck reconstruction after (chemo)radiation: facts and prejudices. Curr Opin Otolaryngol Head Neck Surg 2016;24:83-90. 